\title{
INVESTIGACIÓN
}

Recibido: 12/03/2021 --- Aceptado: 15/04/2021 --- Publicado: 24/05/2021

\section{LA MEDICIÓN DE LAS ESTRATEGIAS DE MARKETING EXPERIENCIAL PARA LA GENERACIÓN MILLENNIAL EN EL SECTOR HOTELERO LEISURE ESPAÑOL}

\section{The measurement of experiential marketing strategies for millennials in the Spanish leisure hotel sector}

Laura Benítez-Coll: Universidad Internacional de La Rioja. España. laura.benitez.coll@gmail.com

Eduardo Barrio-Menoyo: Universidad Internacional de La Rioja. España. edubmh@gmail.com

Como citar el artículo:

Benítez-Coll, L. y Barrio-Menoyo, E. (2021). La medición de las estrategias de marketing experiencial para la generación millennial en el sector hotelero Leisure español. Vivat Academia. Revista de Comunicación, 154, 343-360. http://doi.org/10.15178/va.2021.154.e1355

\section{RESUMEN}

El objetivo de este estudio es comprobar si las estrategias de marketing experiencial y experiencia del cliente aplicadas en los hoteles son factores diferenciales para atraer al segmento millennial a dichos establecimientos, así como también saber si son exitosas y rentables. Para ello, se ha realizado, en primer lugar, una investigación sobre el comportamiento del público objetivo elegido cuando selecciona un alojamiento para sus vacaciones, con la finalidad de descubrir sus insights y los constructos básicos más valorados por ellos y que nos permitirán, posteriormente, conocer la rentabilidad de las estrategias aplicadas. Para dicha investigación, se ha seguido un enfoque neurocualitativo complementado por una técnica cuantitativa. Así, para analizar los insights del segmento millennial y definir al buyer persona se han combinado las siguientes técnicas: encuesta semiestructurada CAWI, utilizando el muestreo aleatorio simple, focus group y test de asociación implícita. Además, se han realizado entrevistas semiestructuradas en profundidad a diferentes figuras del sector hotelero para conocer su opinión sobre el uso del marketing experiencial en sus planes de negocio y sus consideraciones con respecto al segmento millennial. Con esta información, se ha llevado a cabo un análisis multifactorial del concepto de "experiencia”, utilizando los constructos descubiertos en la investigación. 
Benítez-Coll, L. y Barrio-Menoyo, E.

La medición de las estrategias de marketing experiencial para la generación millennial en el sector hotelero Leisure español

PALABRAS CLAVE: Millennial - Marketing experiencial - Comportamiento del consumidor - Insights - Neuromarketing - Hoteles - Experiencia del cliente

\begin{abstract}
The aim of this research is to check whether the experiential marketing and customer experience strategies implemented by hotels constitute differential factors to attract the millennial segment to their accommodation, as well as to determine up to what extent these strategies are successful and profitable. In order to fulfil this purpose, different research and surveys have been carried out. In the first place, we led a behavioural research of the target when choosing accommodation for their holidays. The purpose of this research was to find out the target's insights as well as the constructs they appreciate most, in order to later determine the cost-effectiveness of the strategies used. The approach was neuroqualitative, however supplemented with a quantitative technique. So as to analyse the insights of the millennial segment, as well as to define the buyer persona, a CAWI semi-structured survey (simple random sampling) was used, with the aid of the following techniques: focus group and an implicit association test. Also different personalities from the hotel sector were interviewed in depth by means of semi-structured interviews so as to look into their opinions about the use of experiential marketing in their business plans, in regard to the millennial segment. Once gathered this information we have performed a multifactorial analysis of the concept "experience", making use of the constructs discovered in our research.
\end{abstract}

KEY WORDS: Millennial - Experiential marketing - Consumer behavior - Insights Neuromarketing - Hotels - Customer experience.

\title{
1. INTRODUCCIÓN
}

El presente trabajo se centra en el estudio del uso del marketing experiencial en el sector hotelero vacacional español, principalmente enfocado en el segmento millennial.

La razón para elegir este sector del mercado ha sido debido al enorme impacto que tienen este tipo de estrategias en el sector turístico. Dentro de él, se ha escogido el ámbito de la hostelería pues el sector turístico es demasiado amplio para estudiarlo en su totalidad y, por tanto, resulta mucho más práctico centrarse en un sector específico, como los hoteles, que suelen utilizar este tipo de estrategias en sus negocios. "El sector hotelero comprende todos aquellos establecimientos que se dedican profesionalmente a proporcionar alojamiento con o sin servicios integrados por una contraprestación económica, prestando servicios heterogéneos, combinables e integrables en su mayoría" (Escamilla y Duque, 2011).

De la misma forma, como se indica en el estudio de Williams, Castañeda, Galindo y Martínez (2018), el sector vacacional es el más dispuesto para la vivencia de nuevas

Vivat Academia. Revista de Comunicación. 2021, nº 154, 343-360 
Benítez-Coll, L. y Barrio-Menoyo, E.

La medición de las estrategias de marketing experiencial para la generación millennial en el sector hotelero Leisure español

emociones y experiencias, por lo que hacer uso de estrategias de marketing experiencial en este sector resulta idóneo.

A su vez, la razón por la que nos hemos enfocado en el segmento millennial es debido a que ellos son los primeros que se supone que valoran las experiencias por encima del precio a la hora de consumir.

Las hipótesis a las que buscamos dar respuesta mediante esta investigación son, averiguar si las estrategias de marketing experiencial aportan una diferenciación de la competencia que genere una mayor satisfacción en el consumidor al superar sus expectativas y que, de este modo, se genere una mayor lealtad a la marca y una mayor recomendación por parte de los clientes.

Hoy en día, el marketing experiencial ha cobrado una gran importancia en el mercado. Los métodos tradicionales de marketing en los que los objetivos eran lograr una mayor producción y satisfacer únicamente las necesidades fisiológicas de los compradores han quedado atrás debido a que el consumidor actual está mucho más informado sobre todas las alternativas de productos disponibles a los que puede acceder, de tal forma que ya no busca un producto que satisfaga sus necesidades, sino un producto que le ofrezca algo más (una experiencia) y que se diferencie de la competencia (Freund, 2016).

El concepto de experiencia nace en los años 60 (Castañeda, Galindo, Martínez, 2018) y se define, según Casado y Merino (2011, p. 63), como “un suceso privado que se produce como respuesta a una estimulación. No se autogenera, sino que es inducida por algo o alguien externo".

Se puede decir que el uso de las experiencias en los hoteles empieza en el s. XIX cuando César Ritz introduce en sus establecimientos, "habitaciones con baño, una restauración cuidada y un servicio de atención al cliente personalizado" (Caridad y Núñez, 2016).

"La experiencia juega un importante papel ya que la imagen que provee genera un cambio positivo o negativo en el consumidor" (Fatma, 2014, p. 42). La experiencia del consumidor con el producto o servicio resulta en una satisfacción que deriva en la lealtad del consumidor al producto. "La experiencia pasa a ser un producto comercial cuando se vuelve memorable para los consumidores" (Freund, 2016).

Debido a la importancia que tiene generar experiencias positivas en los consumidores debemos tener claro cuáles son aquellas experiencias que generan satisfacción. Las experiencias eficaces son aquellas que surgen por la interacción con distintas situaciones memorables que inciden tanto en nuestros pensamientos, como en nuestros sentimientos y sensaciones.

"La experiencia se ve como un evento privado que surge como respuesta a un estímulo" (Crouse, 2010, p. 18). "Cuando el individuo se enfrenta a estímulos en el

Vivat Academia. Revista de Comunicación. 2021, n 154, 343-360 
Benítez-Coll, L. y Barrio-Menoyo, E.

La medición de las estrategias de marketing experiencial para la generación millennial en el sector hotelero Leisure español

ambiente de compra, están viviendo una experiencia de consumo" (Crouse, 2010, p. 18). Diseñar una experiencia positiva requiere una comprensión de en qué consiste una experiencia y cuáles son sus dimensiones y componentes.

En base a esta información "una experiencia memorable se puede definir como el cambio comportamental, emocional, sensorial, cognitivo o relacional con los valores funcionales en las áreas cerebrales neurobiológicas generadas por las sensaciones y emociones del ambiente físico" (Guzel y Taylan, 2015).

Para poder realizar esta investigación nos hemos basado en el modelo de O'Sullivan y Spangler para la creación de estrategias de marketing experiencial. Este modelo analiza la experiencia en base a las emociones y acontecimientos ocurridos antes, durante y después de esta. La experiencia también es analizada según los factores y valores que generan participación y valor a la misma, según las necesidades de la experiencia que generan participación, el papel que desempeñan los participantes en la experiencia en base a su personalidad y el panel de proveedores de la experiencia, es decir, la capacidad de estos para personalizar, controlar y coordinar la experiencia.

Las estrategias utilizadas en el marketing experiencial se basan en el concepto de customer centric, esto es, crear toda la estrategia de marca poniendo al consumidor y su comportamiento en el centro de esta o "centrarse en cómo experimentan las marcas los consumidores, no cómo se perciben" (Lenderman, 2008). Además, las experiencias creadas deben tener en cuenta todas las interacciones que el cliente tiene con la marca en todos los momentos de contacto, antes, durante y después de la compra, tratando de dar vida a las promesas que hace a sus consumidores (Lenderman, 2008). Es decir, este tipo de estrategias se centran en que el consumidor obtenga una experiencia global satisfactoria y no solo un servicio que aporte lo esperado por el público.

En el sector hotelero el uso del marketing experiencial adquiere mucha importancia debido a que la estancia en un hotel es algo muy personal y, por ello, se deben cuidar al detalle todas las características que ofrece para que así la experiencia global del cliente sea lo más positiva posible y genere un buen recuerdo de su estancia con una mayor probabilidad de repetir la experiencia en el futuro y de recomendarla a personas de su entorno.

Para todo ello, el conocimiento del cliente es fundamental y el neuromarketing resulta una herramienta muy útil para adquirir tal conocimiento. El neuromarketing supone una revolución al no depender de declaraciones verbales y al aportar una información implícita sobre los consumidores que no puede ser sesgada, es decir, nos aporta un tipo de conocimiento más objetivo. Mediante el neuromarketing podemos explorar el comportamiento humano desde una perspectiva cerebral, utilizando las neurociencias o como define Morin (2011), el "neuromarketing es al marketing lo que la neuropsicología a la psicología". 
Benítez-Coll, L. y Barrio-Menoyo, E.

La medición de las estrategias de marketing experiencial para la generación millennial en el sector hotelero Leisure español

Mediante este tipo de estrategias de neuromarketing se podrá analizar hasta qué punto se ven atraídos realmente los consumidores millennials por el uso del marketing experiencial durante la estancia elegida en sus viajes.

\section{OBJETIVOS}

El objetivo principal de esta investigación es identificar cuáles son los touchpoints de mayor importancia en el customer journey map de la experiencia vivida para la generación millennial, de modo que al identificarlos se pueda proporcionar una experiencia totalmente satisfactoria para este segmento de la población.

Los objetivos secundarios, por otra parte, son:

1. Medir cuál es la eficiencia del marketing experiencial para atraer a la generación millennial.

2. Analizar cuáles son las necesidades y prejuicios que presenta el uso del marketing experiencial en hoteles en la actualidad realizando entrevistas a distintas figuras del sector hotelero.

3. Detectar qué métodos de medición existen actualmente que nos permitan analizar los resultados obtenidos en las estrategias de marketing experiencial.

4. Descubrir qué características son las que definen las experiencias que los millennials valoran por encima del precio durante la estancia en sus viajes vacacionales. Al tener claro cómo son este tipo de experiencias sabremos cómo atraer mejor a este público.

\section{METODOLOGÍA}

La investigación se ha diseñado con un enfoque principalmente neurocualitativo con una finalidad de tipo exploratorio-descriptivo, para conocer los insights, tanto del cliente millennial como de las empresas de hoteles. Complementariamente, se ha utilizado la encuesta CAWI (Computer Aided Web Interviewing) como complemento cuantitativo para obtener información de una muestra mayor. La investigación tiene las siguientes características:

- Grado de control: observacional

- Recopilación de la información: retrospectiva (la información utilizada para el marco teórico ya es existente y es utilizada para generar una información nueva)

- Secuencia temporal: transversal

La población muestral millennial en España es de 8.391 .713 habitantes, según datos del padrón del INE a 1 de enero de 2020. En febrero de 2020, antes del inicio de la pandemia y según datos del INE, había en España 12.662 establecimientos hoteleros abiertos.

La población muestral elegida debía contar con las siguientes características:

- Personas de cualquier género 
Benítez-Coll, L. y Barrio-Menoyo, E.

La medición de las estrategias de marketing experiencial para la generación millennial en el sector hotelero Leisure español

- Nacidos entre 1981 y 1996

- Residentes en España

- Clase media amplia

- Estudios superiores

- Destinan parte de sus vacaciones y recursos a viajar

- Viajar es una parte importante de sus vidas

- Deben buscar un alojamiento para sus vacaciones en el destino elegido.

La población muestral utilizada en la encuesta CAWI se seleccionó por medio de un muestreo aleatorio simple, mientras que los participantes en las demás técnicas se eligieron en base a las respuestas dadas en la encuesta CAWI habiendo respondido de forma correcta a las preguntas que contaban como variables de control.

Con las técnicas utilizadas se ha querido conocer en profundidad los insights y necesidades del segmento millennial para poder realizar una propuesta basada en la realidad observada y no solamente en la teoría de la revisión bibliográfica. Las técnicas utilizadas han sido las siguientes:

\subsection{Investigación segmento millennial}

Objetivo: Con estas técnicas neuro-cuali-cuanti se ha querido dar respuesta a los objetivos relacionados con los touchpoints y elementos y experiencias valoradas por el segmento.

\subsubsection{Focus group}

Se organizaron dos focus group con 8 personas de la generación millennial cada uno, divididas según el género y la edad (nacidos entre 1981-1988 - en adelante, analógicos - y nacidos entre 1989 y 1996 - en adelante, digitales). De esta forma, los grupos se formaron con: 4 mujeres, 2 analógicas y 2 digitales y 4 hombres, 2 analógicos y 2 digitales.

Objetivo: conseguir la información necesaria para determinar qué experiencias y elementos son más valorados por los millennials durante sus estancias en hoteles y qué elementos mejoran su valoración, lealtad y recomendación. Así, se han querido descubrir sus insights a la hora de escoger un hotel de vacaciones y descubrir su customer journey map ideal.

\subsubsection{Encuestas CAWI}

Se ha diseñado una encuesta CAWI semiestructurada, programada en Google Forms y distribuida por Internet siguiendo el muestreo aleatorio simple a 152 personas. 
Benítez-Coll, L. y Barrio-Menoyo, E.

La medición de las estrategias de marketing experiencial para la generación millennial en el sector hotelero Leisure español

El objetivo inicial era estratificar en base a grupos de edad (analógicos y digitales) y género, para observar las posibles diferencias. Lo ideal para este estudio habría sido contar con la muestra suficiente para ello. Sin embargo, debido a las limitaciones para conseguir la muestra necesaria, como el tiempo para el desarrollo del estudio o el acceso a suficientes participantes, el estudio se ha limitado a un análisis global de la muestra.

Para el cálculo y análisis de resultados se ha utilizado el programa SPSS, las herramientas ofrecidas por Google Forms y nubes de palabras, para identificar palabras repetidas en las preguntas abiertas, difícilmente codificables. Se pueden observar detalles diferenciadores por género y edad que deberían confirmarse con una muestra mayor.

Objetivo: obtener información sobre gustos y hábitos de los millennials en hoteles.

\subsubsection{Test de Asociación Implícita (TAI)}

Se ha realizado a 32 personas para comprobar las posibles vinculaciones entre los conceptos "experiencia" y "servicio" y los atributos "positivo" y "negativo" en esta generación. El resultado esperado con esta prueba es que "experiencia" y "positivo" presentara una correlación alta, lo que indicaría que la actitud de los millennials hacia las experiencias es más positiva que la que tienen hacia los servicios de un hotel.

El programa utilizado para realizarlo es I+IAT Management

\subsection{Investigación sector hotelero}

Los objetivos relacionados con las necesidades de los hoteleros respecto las estrategias de marketing experiencial y su medición se contestaron mediante entrevistas semiestructuradas en profundidad a diferentes representantes del sector. Se redactó un guion con distintas preguntas de interés para la investigación, pero también se dejó fluir la conversación según la información aportada por cada entrevistado, siempre que se respondieran las preguntas clave.

Objetivo: obtener información sobre qué estrategias de customer experience se están utilizando y cómo las valoran, en qué se basan las experiencias que ofrecen, qué opinión tienen sobre el uso del marketing experiencial en sus establecimientos $\mathrm{y}$, sobre todo, cómo hacen la medición de resultados.

\section{RESULTADOS}

A continuación, se muestran los resultados generales del estudio:

El precio es la variable más importante en la elección de un hotel y, aunque el alojamiento suele ser una de las partidas más elevadas dentro de un viaje, la percepción es que prefieren gastar el dinero fuera del hotel. Este factor se puede 
Benítez-Coll, L. y Barrio-Menoyo, E.

La medición de las estrategias de marketing experiencial para la generación millennial en el sector hotelero Leisure español

deber a que la mayoría de los participantes recién han acabado sus estudios y empezado a trabajar. Como tienen pocos ingresos prefieren buscar un viaje que sea económico a pesar de que la experiencia ofrecida por el hotel no sea tan buena como en otras opciones. Esto no significa que no den importancia a la experiencia, sino que prefieren utilizar sus recursos fuera del hotel.

En general, se asocia el concepto de experiencia al de actividad y, cuando se les pregunta, tienen un concepto anticuado de lo que se puede realizar en el hotel, por ejemplo, la típica cena con espectáculo, o también asociando las experiencias que ofrece un hotel como demasiado turísticas.

Así, se detecta una oportunidad para el sector hotelero para conocer muy bien a su cliente y especializarse en experiencias que sean vistas como únicas. El reto será el mensaje pues, en general, la opinión mayoritaria es que no valoran las experiencias facilitadas en los hoteles. Se ha realizado un pequeño estudio sociológico con las preguntas abiertas de la encuesta. En cierta manera, estos resultados coinciden con lo manifestado por los participantes de los Focus Group. Cuando se les preguntó qué factor es más importante para ellos a la hora de elegir un hotel, si las experiencias y el trato ofrecidos o el precio, el 76,3\% de los encuestados eligieron el precio. Cuando se les preguntó "por qué", se producen dos razones principalmente: aunque depende mucho del tipo de viaje, muchos asocian el hotel solamente como un lugar donde pernoctar. Otros, en cambio, aducen a los trabajos precarios como motivo para elegir hoteles baratos, argumentando que, si no, no pueden viajar.

Según los comentarios de los millennials encuestados se percibe que, en general, prefieren buscar las experiencias fuera del hotel, viéndolo únicamente como un lugar de descanso. Solo elegirá un hotel que les proporcione una experiencia única, en cuanto a actividades, si realmente es algo que sea de su interés y no puedan encontrarlo en otro sitio, siendo el trato recibido y los consejos por parte del personal del hotel, muy importantes cuando ya han llegado allí. Así, para la elección se rigen por los comentarios de otros huéspedes antes de realizar la reserva.

Sin embargo, incidir en marketing experiencial que facilite la visita y la haga más cómoda utilizando herramientas que hagan más eficiente el trato con el cliente, sin aumentar demasiado el precio, puede ser el factor diferencial que tengan los hoteles, teniendo en cuenta que los aspectos a los que más importancia dan los millennials al buscar un hotel, además del precio, son: localización, trato recibido, eficacia del personal del hotel para responder a sus demandas y limpieza.

Estos resultados son así tanto en la encuesta como en los distintos Focus Group. 
Benítez-Coll, L. y Barrio-Menoyo, E.

La medición de las estrategias de marketing experiencial para la generación millennial en el sector hotelero Leisure español

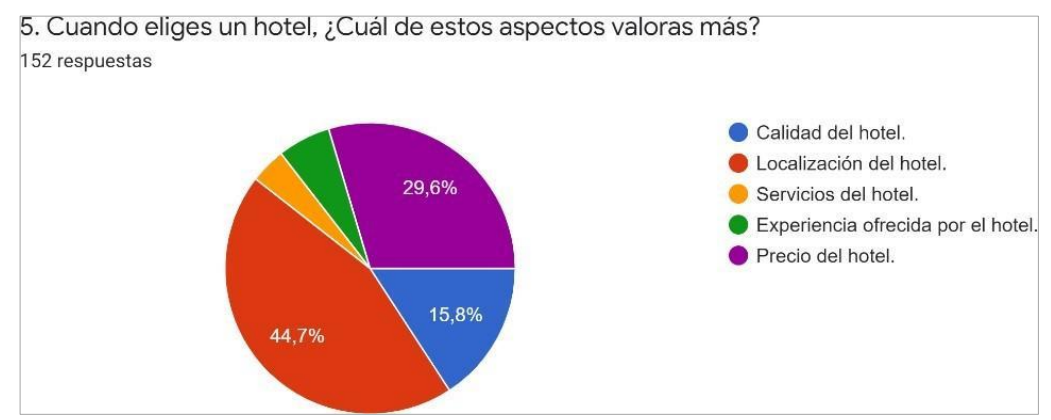

Gráfico 1: Gráfico circular respuesta encuesta CAWI ¿Cuál de estos aspectos valoras más?

Fuente: Elaboración propia basado en formulario de Google Forms

Algunos participantes indican que, para eventos especiales, como un aniversario de boda, sí que se fijan más en la experiencia ofrecida y podrían llegar a pagar un poco más. También se ha podido comprobar que, cuando se trata de distintas opciones con precios similares, entonces los consumidores eligen en función de la experiencia y no del precio.

La generación millennial prefiere hacer sus reservas y buscar información para su viaje por Internet y medios electrónicos, además de hacer uso de buscadores especializados para viajes como Tripadvisor o Booking.com.

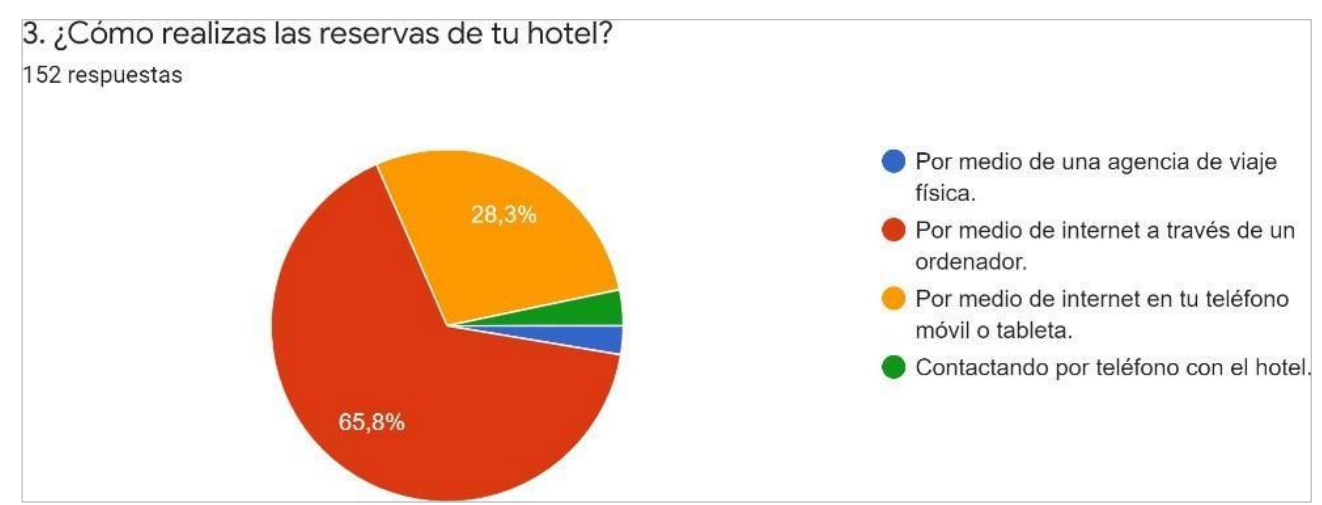

Gráfico 2: Gráfico circular respuesta encuesta CAWI ¿Cómo realizas las reservas de tu hotel?

Fuente: Elaboración propia basado en formulario de Google Forms

Los resultados obtenidos con los Focus Group confirman que los consumidores millennials realizan sus reservas y la búsqueda de información del hotel por internet con dispositivos móviles, ya sea haciendo uso de metabuscadores o mediante la página web oficial del hotel. 
Benítez-Coll, L. y Barrio-Menoyo, E.

La medición de las estrategias de marketing experiencial para la generación millennial en el sector hotelero Leisure español

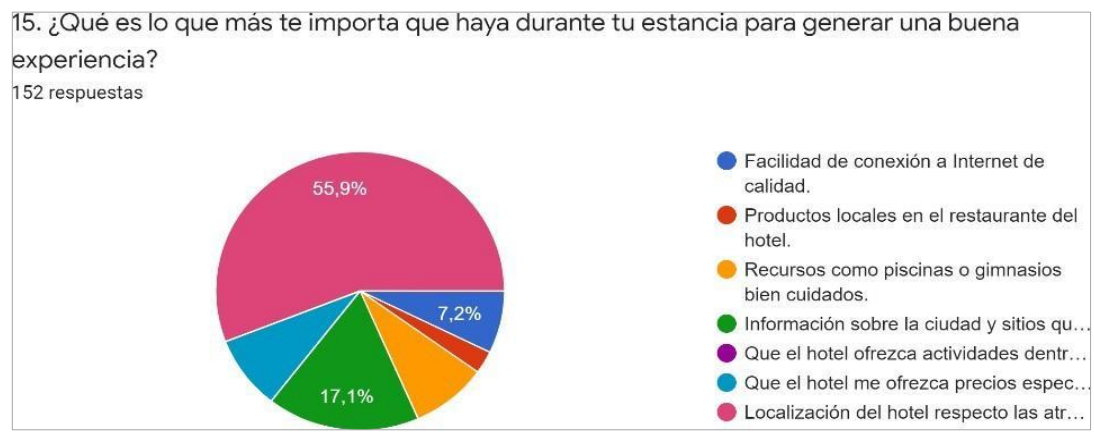

Gráfico 3: Gráfico circular respuesta encuesta CAWI ¿Qué es lo que más te importa que haya durante tu estancia para generar una buena experiencia?

Fuente: Elaboración propia basado en formulario de Google Forms

En los resultados obtenidos, las respuestas de los participantes no cambian en función del sexo o edad. Sin embargo, para obtener unos resultados más fiables, en futuros estudios la muestra deberá ser más amplia y contar con una cantidad equivalente de participantes de cada rango de edad y género.

La forma de ver los hoteles es distinta en los participantes. Mientras algunos ven un hotel como una zona de ocio y entretenimiento, otros solo lo ven como una zona de descanso, pero también depende un poco del tipo de viaje.

Los factores a los que más importancia dan los millennials al instalarse en un hotel son el trato del servicio, la limpieza, el acceso a internet y la localización del hotel.

Para realizar su reserva, la mayoría de los participantes sigue los pasos indicados por la página web que estén utilizando (buscando en distintos metabuscadores, adaptando el viaje a sus necesidades, consultando reseñas de otros usuarios...).

Para la mayoría de los participantes una experiencia maravillosa en un hotel se consigue en base a los servicios que éste oferta y al trato recibido por el personal del hotel, pero su grado de repetición de la experiencia se basa prácticamente en las ofertas que el consumidor pueda encontrar.

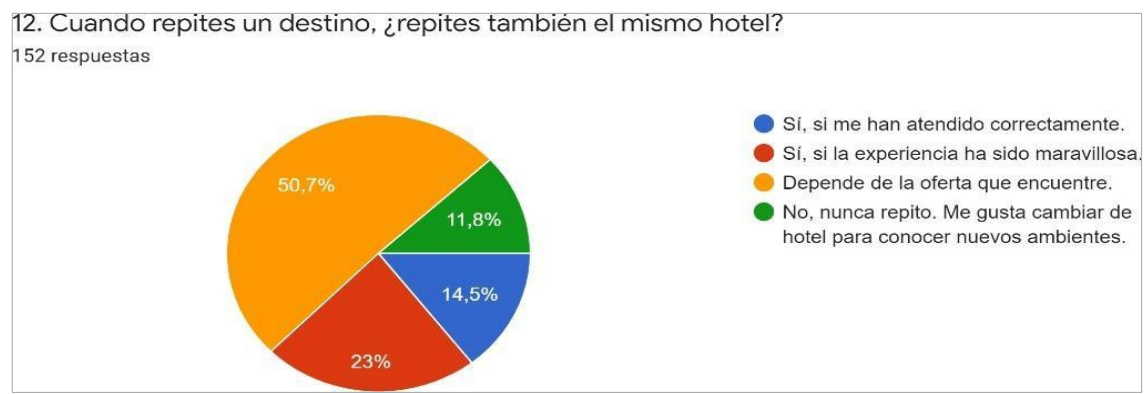

Gráfico 4: Gráfico circular respuesta encuesta CAWI: Cuando repites un destino, ¿repites también el mismo hotel?

Fuente: Elaboración propia basado en formulario de Google Forms 
Benítez-Coll, L. y Barrio-Menoyo, E.

La medición de las estrategias de marketing experiencial para la generación millennial en el sector hotelero Leisure español

Se ha podido observar que la mayoría de los participantes dan importancia a las experiencias negativas en los hoteles, pues, en este caso, tienen claro que no repetirían en ese hotel. Asimismo, la mayoría ve necesario hacer uso del marketing experiencial en los hoteles.

En resumen, los consumidores deciden repetir la estancia en un hotel si el trato recibido ha sido excelente y si el hotel les ha ofrecido una experiencia que ha superado sus expectativas. Si se tiene en cuenta el efecto de aversión al riesgo: los consumidores prefieren repetir la experiencia en un sitio que saben que les aportará una experiencia positiva que en un lugar desconocido. En este punto, los hoteles tienen una oportunidad para superar las expectativas del cliente, puesto que, en la actualidad, dichas expectativas en cuanto a experiencias esperadas no son muy elevadas.

Todos los participantes dan mucha importancia a contar con reseñas y opiniones de otras personas para elegir su estancia. Además, se da más importancia a las opiniones de semejantes lo que nos indica el efecto de búsqueda de confort social. Este mismo fenómeno lo podemos observar en base a las respuestas de esta gráfica, donde se indica el momento en el que los consumidores se decantan por un hotel.

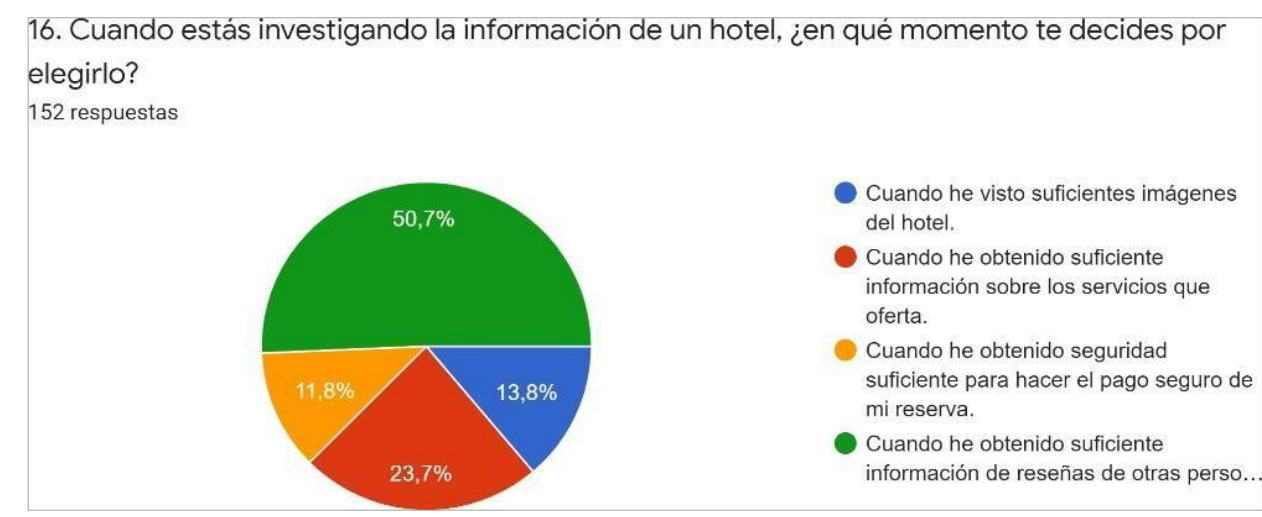

Gráfico 5: Gráfico circular respuesta encuesta CAWI - Cuando estás investigando la información de un hotel, ¿en qué momento decides elegirlo?

Fuente: Elaboración propia basado en formulario de Google Forms

En caso de tener algún problema, tanto en la reserva como en la estancia, la mayoría de los participantes prefieren tener contacto humano para solucionarlo. Aunque les gusta que los procesos sean automáticos, en caso de error, prefieren solventarlo mediante contacto humano. 
Benítez-Coll, L. y Barrio-Menoyo, E.

La medición de las estrategias de marketing experiencial para la generación millennial en el sector hotelero Leisure español

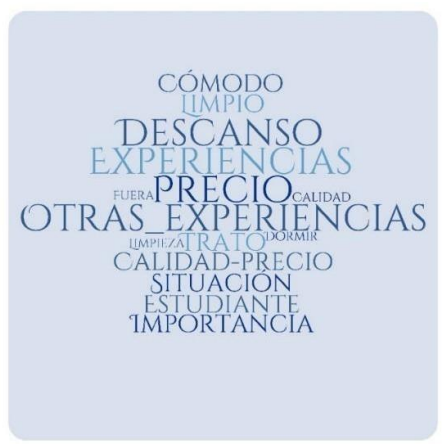

Gráfico 6: Nube de palabras del análisis de la pregunta abierta “Qué crees que resulta más importante: el precio a pagar o la experiencia que ofrecen y trato recibido y por qué?"

Fuente: www.nubedepalabras.es con datos extraídos de la encuesta CAWI

Gráfico 7: Nube de palabras del análisis de la pregunta abierta "Experiencias positivas y negativas vividas por los encuestados en hoteles"

Fuente: wwW.nubedepalabras.es con datos extraídos de la encuesta CAWI

En cuanto a los datos obtenidos mediante el TAI, con una muestra de 33 personas (12 hombres y 21 mujeres, 21 analógicos y 12 digitales), en general, se da una asociación ligera entre experiencias y positivo, y servicios y negativo, por lo que no se puede confirmar la hipótesis inicial de que los millennials tienen actitudes más positivas respecto las experiencias que los servicios cuando se trata de servicios de alojamiento.

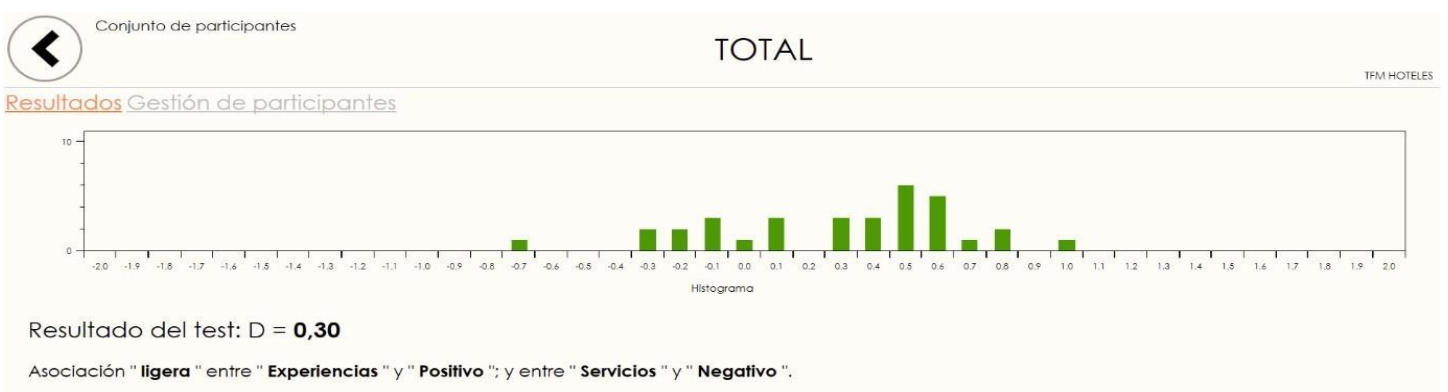

Gráfico 9: Resultado TAI del conjunto de participantes "Total" Fuente: I+AIT Interactúa+ con datos extraídos del Test de Asociación Implícita 
Benítez-Coll, L. y Barrio-Menoyo, E.

La medición de las estrategias de marketing experiencial para la generación millennial en el sector hotelero Leisure español

Al comparar por género vemos que esta asociación es bastante más alta en mujeres que en hombres.

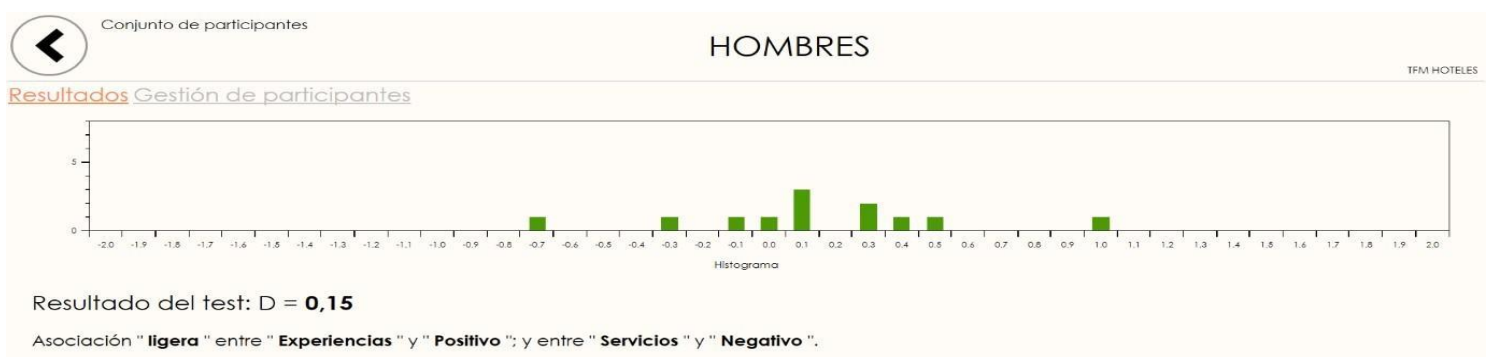

Gráfico 10: Resultado TAI del conjunto de participantes "Hombres" Fuente: I+AIT Interactúa+ con datos extraídos del Test de Asociación Implícita

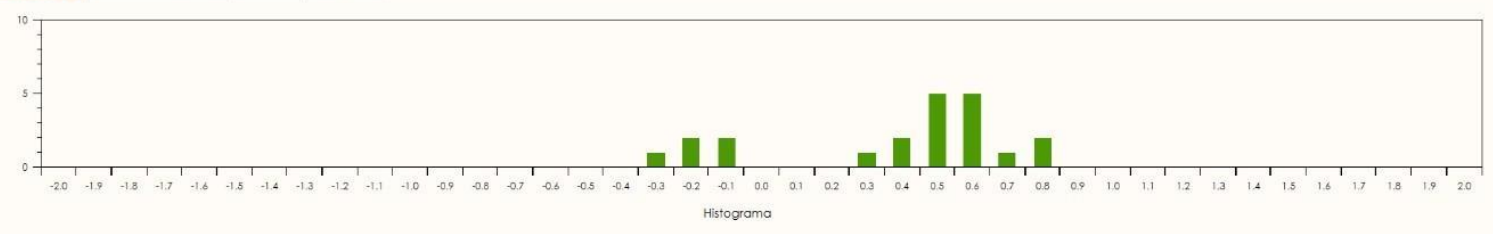

Resultado del test: $D=\mathbf{0 , 3 9}$

Asociación "moderada "entre " Experiencias "y " Positivo "; y entre " Servicios "y " Negativo ".

Gráfico 11: Resultado TAI del conjunto de participantes "Mujeres" Fuente: I+AIT Interactúa+ con datos extraídos del Test de Asociación Implícita

Al comparar por grupos de edad vemos que apenas se genera diferencia.

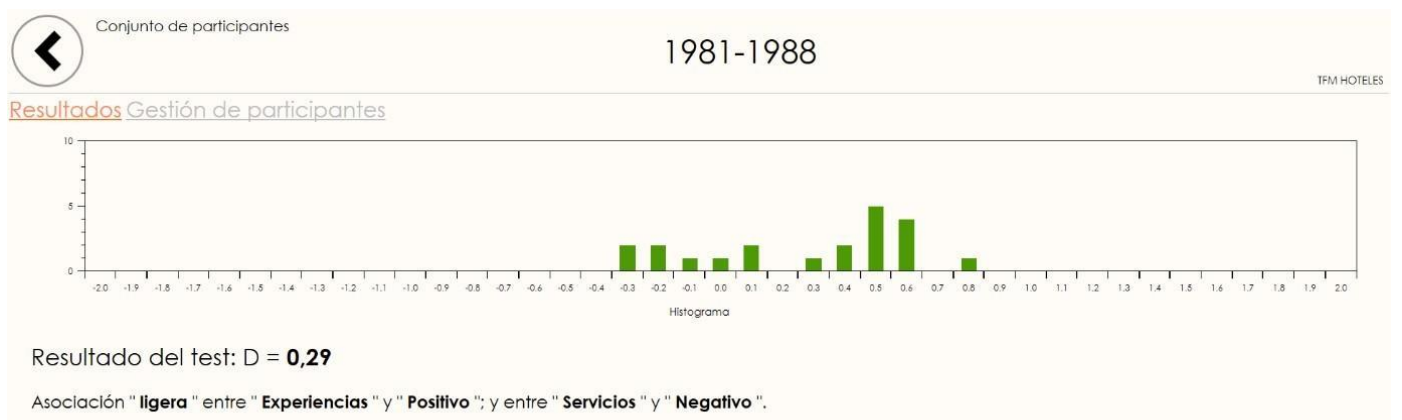

Gráfico 12: Resultado TAI del conjunto de participantes "Nacidos entre 1981-1988" Fuente: I+AIT Interactúa+ con datos extraídos del Test de Asociación Implícita 
Benítez-Coll, L. y Barrio-Menoyo, E.

La medición de las estrategias de marketing experiencial para la generación millennial en el sector hotelero Leisure español

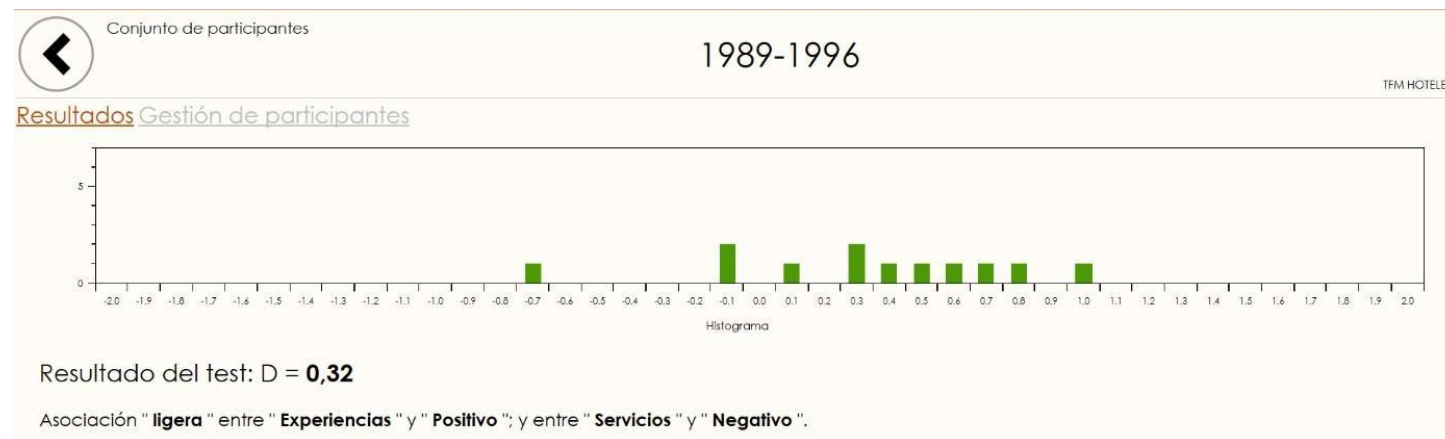

Gráfico 13: Resultado TAI del conjunto de participantes "Nacidos entre 1989-1996"

Fuente: I+AIT Interactúa+ con datos extraídos del Test de Asociación Implícita

Las entrevistas en profundidad se han realizado principalmente a directores de marketing $\mathrm{o}$ directores $\mathrm{y}$ propietarios de hoteles de cadenas pequeñas, independientes, familiares... En futuras investigaciones, sería interesante comparar sus respuestas con las de responsables de las grandes cadenas hoteleras que normalmente cuentan con su propio departamento de customer experience, para observar las diferencias entre ambas tipologías de management.

En general, los entrevistados consideran que el segmento millennial es muy interesante porque marcará el futuro del alojamiento con sus hábitos de consumo. Algunos no tienen prácticamente contacto con ellos y otros han empezado a implementar estrategias para captarlos, normalmente, sin apostar $100 \%$, sino más bien haciéndoles convivir con otros segmentos.

Todos coinciden en que el marketing experiencial y el customer experience es una buena forma de diferenciarse y creen que al segmento millennial le puede gustar. El tipo de experiencias que se generan para los millennial son: uso de tecnología (check-in online, wearables), cromoterapia, aromaterapia, música cuidada, experiencias gourmet, fiestas temáticas, uso de redes sociales como forma de comunicación con ellos, información de interés inmediata a través del smartphone, skybar, recordando siempre que el elemento visual es uno de los más importantes por el uso de Instagram. Esto implica la necesidad de conocer muy bien al cliente objetivo y darle lo que está buscando mediante una experiencia única e individualizada, esto es, conociendo sus insights y sus pain.

Se ha visto que la mayoría de los hoteleros entrevistados no tienen una segmentación muy clara, lo cual no significa que no haya ejemplos de hoteles que utilicen esta estrategia de forma más eficiente. En todo caso, se puede decir que, aunque su enfoque sea algo más generalista, sí hacen esfuerzos por conocer muy bien a su cliente y consideran que la clave está en la personalización de experiencias y el trato excelente durante la estancia.

En cuanto a sus aplicaciones del marketing experiencial, a prácticamente todos les falta una estrategia clara. Muchos de ellos utilizan el método de "ensayo-error", 
Benítez-Coll, L. y Barrio-Menoyo, E.

La medición de las estrategias de marketing experiencial para la generación millennial en el sector hotelero Leisure español

probando distintas herramientas, manteniendo aquello que funciona y descartando lo que no, basándose en la intuición más que en métricas concretas. Han constatado que funciona y que las ventas han aumentado, pero ninguno de los entrevistados sabe muy bien por qué, $\mathrm{y}$, mucho menos, han conseguido llevarlo a resultados. Algunos de ellos han manifestado que los elementos y software específicos de medición pueden resultar inasumibles para cadenas pequeñas $u$ hoteles independientes.

Básicamente realizan sus mediciones a través de encuestas, bien con los formularios tradicionales, entrevistando a sus clientes o con herramientas más modernas de medición, como la herramienta TrustYou o las encuestas NPS.

La situación cambia un poco si la venta es directa a través de su web, lo que permite también tener mayor capacidad de personalización de la experiencia ya desde la búsqueda de opciones, más difícil si su canal de distribución viene determinado por OTA (Online Travel Agency) y metabuscadores.

Unánimemente, ninguno de ellos realiza una medición del retorno de la inversión de estas estrategias.

\section{DISCUSIÓN}

Las conclusiones más relevantes que podemos extraer de esta investigación son las siguientes:

1. Al valorar la experiencia global del viaje, vemos que los participantes sí valoran las experiencias vividas en el destino, por lo que invierten gran parte de su presupuesto en ellas. En base a esta información concluimos que los usuarios sí valoran las estrategias de marketing experiencial, aunque el precio sigue recibiendo gran importancia ya que es el factor definitivo para poder permitirse o no la experiencia y para elegir un hotel.

2. Los hoteles se ven como zonas de descanso más que como lugares de experiencias. Los participantes siguen considerando el uso de experiencias en hoteles de una forma anticuada y no como una experiencia novedosa e individualizada en base a sus gustos y necesidades.

3. Los consumidores consideran una experiencia perfecta cuando se les ofrece un trato percibido como excelente y cuando los servicios del hotel han sido de calidad. En base a esto se concluye que los touchpoints que más importancia adquieren en el customer journey map del hotel son aquellos que ocurren dentro del mismo hotel, es decir, los servicios y personal que provee el hotel y sobre los que tiene control.

4. El segmento millennial presenta una serie de insights a los que los hoteles deben atender para poder captar su atención. Entre los distintos recursos a los que pueden acceder los hoteles para atraer a este público se encuentran el hacer uso de avances tecnológicos e internet en el hotel, ofrecer experiencias 
Benítez-Coll, L. y Barrio-Menoyo, E.

La medición de las estrategias de marketing experiencial para la generación millennial en el sector hotelero Leisure español

diferenciadas y únicas, disponer de personal cualificado y atento a las necesidades de los consumidores, utilizar una decoración llamativa y ofrecer experiencias que superen las expectativas de los clientes y que, al mismo tiempo, sean experiencias acordes a lo que el consumidor espera recibir.

5. Los hoteles dentro de la experiencia global del viaje son vistos como algo intercambiable, incluso como un commodity. Debido a ello, los consumidores suelen comparar hoteles centrándose solo en características comunes como la localización, la limpieza y el buen trato recibido por el personal del hotel, basándose en los comentarios de otros huéspedes.

6. Podemos concluir que el uso de customer experience resulta útil para atraer al segmento millennial, aunque mantener la fidelidad de estos consumidores supone un reto ya que mostrarán una mayor preferencia por obtener ofertas que les permitan viajar y vivir nuevas experiencias que por repetir la misma experiencia en el mismo hotel.

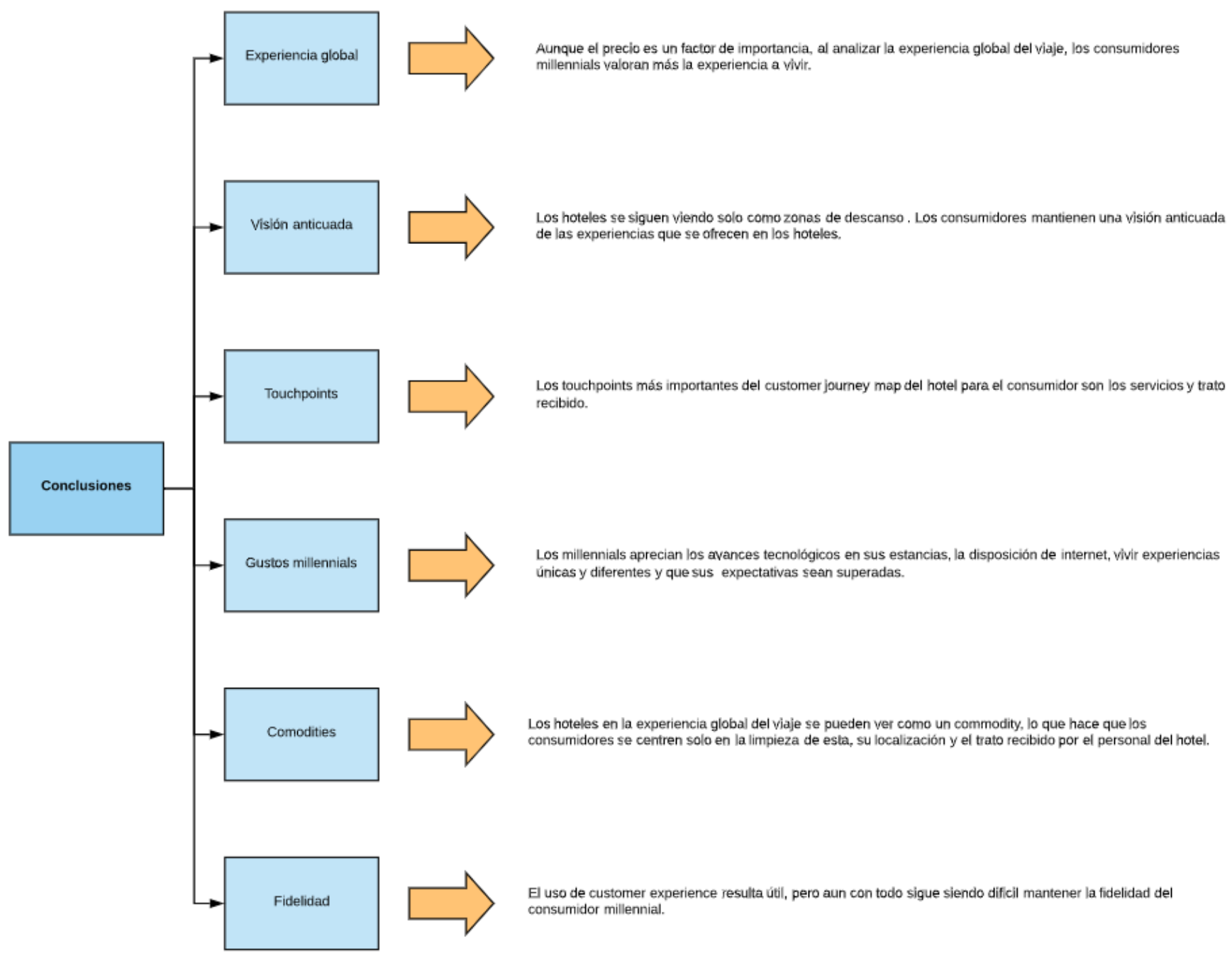

Foto 1: Esquema conclusiones del estudio

Fuente: Elaboración propia 
Benítez-Coll, L. y Barrio-Menoyo, E.

La medición de las estrategias de marketing experiencial para la generación millennial en el sector hotelero Leisure español

\section{REFERENCIAS}

Akdeniz University, Tourism Faculty, Antalya/Turkey, Guzel, O., Dortyol, T., \& Akdeniz University, Ayse Sak School of Applied Sciences, Antalya/Turkey. (2016). Exploring the Multi-Sensory Based Memorable Tourism Experiences: A Study of Adam\&Eve Hotel in Turkey. Journal of Marketing and Consumer Behaviour in Emerging Markets, 2(4), 28-39. https://doi.org/10.7172/24496634.jmcbem.2016.2.2

Caridad, L. y Núñez, J. M. (2016). Desarrollo de la industria hotelera española: el grupo Melià. International Journal of Scientific Management and Tourism. 2(4), 9-37. ISSN-e: 2386-8570, ISSN:

https:// dialnet.unirioja.es/servlet/catart?codigo $=5744218$

2444-0299.

Casado. J. C. y Merino, M. J. (2011). Comunicación experiencial y sensorial: Algunos ejemplos de aplicación. Harvard Deusto business review, 3751, 62-79. Ediciones Deusto. ISBN:

0210-900X. http:/ / dialnet.unirioja.es/ servlet/articulo?codigo $=3394025$

Castañeda García, J. A., Galindo, A. V. y Martínez, R. (2018). The Effect of online and offline experiential marketing on brand equity in the hotel sector. Spanish Journal of Marketing - ESIC, 22(1), 22-41. Emerald Publishing Limited 2444-9709 http:/ / dx.doi.org/10.1108/SJME-03-2018-003. ISSN-e: 2444-9709, ISSN: 2444-9695.

Crouse, T. (2010). The influence of ambient scent on hotel guests' responses. Concordia University. Quebec, Canadá. ISBN: 978-0-494-70989-4. http://www.worldcat.org/oclc/796917695

Escamilla, M. A. y Duque, E. J. (2011). Revisión conceptual de la lealtad en servicios hoteleros. Criterio libre, 9, 181-202. ISSN: $1900-0642$. https:// doi.org/10.18041/1900-0642/criteriolibre.2011v9n14.1236

Fatma, S. (2014). Antecedents and consequences of customer experience management: A literature review and research agenda. International journal of business and commerce, 3, 32-49. ISSN: 2225-2436. https://www.researchgate.net/publication/319256451_Antecedents_and_Conseq uences_of_Customer_Experience_ManagementA_Literature_Review_and_Research_Agenda

Freund, D. (2004). Seeking the "Ultimate hotel experience". ESADE. https://www.researchgate.net/publication/228585213_SEEKING_THE_ULTIMA TE_HOTEL_EXPERIENCE

Lenderman, M. (2008). Marketing experiencial. La revolución de las marcas. Madrid: ESIC Editorial. ISBN: 978-84-7356-545-5. ISSN: 0124-0099. 
Benítez-Coll, L. y Barrio-Menoyo, E.

La medición de las estrategias de marketing experiencial para la generación millennial en el sector hotelero Leisure español

Morin, C. (2011). Neuromarketing: The New Science of Consumer Behavior. Society, 48(2), 131-135. https:// doi.org/10.1007/s12115-010-9408-1

\section{AUTORES}

\section{Laura Benitez-Coll}

Diplomada en Turismo y licenciada en Administración y Dirección de Empresas y con formación de postgrado en Organización y Dirección de Eventos Corporativos (Universidad de les Illes Balears), como $1^{\text {a }}$ de la promoción, y con máster en Estrategia y Gestión de Destinos Sostenibles (2019, Universitat Oberta de Catalunya), con calificación de Sobresaliente, y máster en Neuromarketing (2020, UNIR), con calificación de 9,68. Líneas de investigación de interés: papel de los eventos como factor estratégico de regeneración urbana y reposicionamiento de destinos, neuromarketing, comportamiento del consumidor, customer experience, consumo ético y sostenible, turismo sostenible, turismo comunitario, estrategias de marketing sensorial, especialmente uso del color.

laura.benitez.coll@gmail.com

Orcid ID: https:// orcid.org/0000-0002-0280-9589

\section{Eduardo Barrio-Menoyo}

Graduado en psicología con mención clínica (2018, Universidad de Zaragoza), con calificación 6,68, y máster en Neuromarketing (2020, UNIR), con calificación de 9,32. Líneas de investigación de interés: psicología social, psicología clínica, neuromarketing, customer experience, comportamiento del consumidor, marketing sensorial, eficacia del mindfulness como factor de mejora de los niveles de ansiedad, depresión y estrés.

edubmh@gmail.com

Orcid ID: https:/ / orcid.org/0000-0003-0475-0469 